\title{
Patterns of Vertebrate Isochore Evolution Revealed by Comparison of Expressed Mammalian, Avian, and Crocodilian Genes
}

\author{
Jena L. Chojnowski · James Franklin · Yoshinao Katsu · Taisen Iguchi • \\ Louis J. Guillette Jr. · Rebecca T. Kimball · Edward L. Braun
}

Received: 19 October 2006/ Accepted: 18 May 2007/Published online: 3 August 2007

(C) Springer Science+Business Media, LLC 2007

\begin{abstract}
Vertebrate genomes are mosaics of isochores, defined as long ( $>100 \mathrm{~kb}$ ) regions with relatively homogeneous within-region base composition. Birds and mammals have more GC-rich isochores than amphibians and fish, and the GC-rich isochores of birds and mammals have been suggested to be an adaptation to homeothermy. If this hypothesis is correct, all poikilothermic (cold-blooded) vertebrates, including the nonavian reptiles, are expected to lack a GC-rich isochore structure. Previous studies using various methods to examine isochore structure in crocodilians, turtles, and squamates have led to different conclusions. We collected more than 6000 expressed sequence tags (ESTs) from the American alligator to overcome sample size limitations suggested to be the fundamental problem in the previous reptilian studies. The alligator
\end{abstract}

Reviewing Editor: Dr. Nicolas Galtier

Electronic supplementary material The online version of this article (doi: 10.1007/s00239-007-9003-2) contains supplementary material, which is available to authorized users.

J. L. Chojnowski $(\bowtie) \cdot J$. Franklin · L. J. Guillette Jr. ·

R. T. Kimball · E. L. Braun

Department of Zoology, University of Florida, 223 Bartram Hall, PO Box 118525, Florida, Gainesville 32611, USA

e-mail: jena@zoo.ufl.edu

Y. Katsu · T. Iguchi

Division of Molecular Environmental Endocrinology, Okazaki Institute for Integrative Bioscience, National Institute for Basic Biology, National Institutes of Natural Sciences,

5-1 Higashiyama, Myodaiji, Okazaki 444-8787, Japan

Y. Katsu · T. Iguchi

Department of Basic Biology, School of Life Science, Graduate University for Advanced Studies, 5-1 Higashiyama, Myodaiji,

Okazaki 444-8787, Japan
ESTs were assembled and aligned with their human, mouse, chicken, and western clawed frog orthologs, resulting in 366 alignments. Analyses of third-codon-position GC content provided conclusive evidence that the poikilothermic alligator has GC-rich isochores, like homeothermic birds and mammals. We placed these results in a theoretical framework able to unify available models of isochore evolution. The data collected for this study allowed us to reject the models that explain the evolution of GC content using changes in body temperature associated with the transition from poikilothermy to homeothermy. Falsification of these models places fundamental constraints upon the plausible pathways for the evolution of isochores.

Keywords Isochore - Genome evolution · Cytosine deamination - GC content - Homeothermy · Poikilothermy

\section{Introduction}

Vertebrate nuclear genomes are characterized by distinct biases in nucleotide composition, with strongly (GC) and weakly (AT) base-pairing nucleotides clustering in long (>100 kb) regions of relatively homogeneous base composition called isochores. Neighboring isochores appear to have relatively sharp boundaries, a feature that originally made it possible to separate and characterize isochores using density gradient ultracentrifugation (Bernardi 2000). The availability of draft genome sequences for model systems (e.g., mouse, chicken, Arabidopsis) has made it possible to define isochores computationally using methods like the traditional sliding-window approach and the $\mathrm{Z}$ curve method (Zhang et al. 2001). The regional GC content of genomes is correlated with many important features 
such as the distribution of genes and repetitive elements (Bernardi 2000; Hackenberg et al. 2005), chromosomal banding (Saccone 1997), and patterns of CpG methylation (Caccio et al. 1997).

The genomes of warm-blooded (more properly, homeothermic) amniotes (birds and mammals) exhibit a distinct genomic heterogeneity in GC content, with some regions as low as $30 \% \mathrm{GC}$ and others as high as $60 \% \mathrm{GC}$ (Bernardi 1995). Conversely, cold-blooded (poikilothermic) vertebrates have been proposed to have a more homogeneous genome (Bernardi and Bernardi 1991), and the poikilotherms that are best characterized from a genomic standpoint (fish and amphibians) generally have lower average GC content than birds and mammals (e.g., most of the Xenopus genome has $<45 \%$ GC; see Bernardi 1995). These data led to the assumption that GC-poor isochores are ancestral and conserved among vertebrates. Two different classes of models have been proposed to explain the origin of GC-rich isochores: those based on selection and those based on mutational patterns combined with the fixation of selectively neutral changes (Fig. 1). The former postulate that GC-rich isochores arose for functional reasons (i.e., natural selection) and the latter postulate the existence of regional mutation biases.

Selectionist and mutationalist models of GC-rich isochore evolution can be placed into a variety of mechanistic categories. Previous analyses of reptilian genome data have addressed the role of thermal factors in isochore evolution (Eyre-Walker and Hurst 2001; Hughes et al. 1999; Varriale and Bernardi 2006), and this study will focus on the division

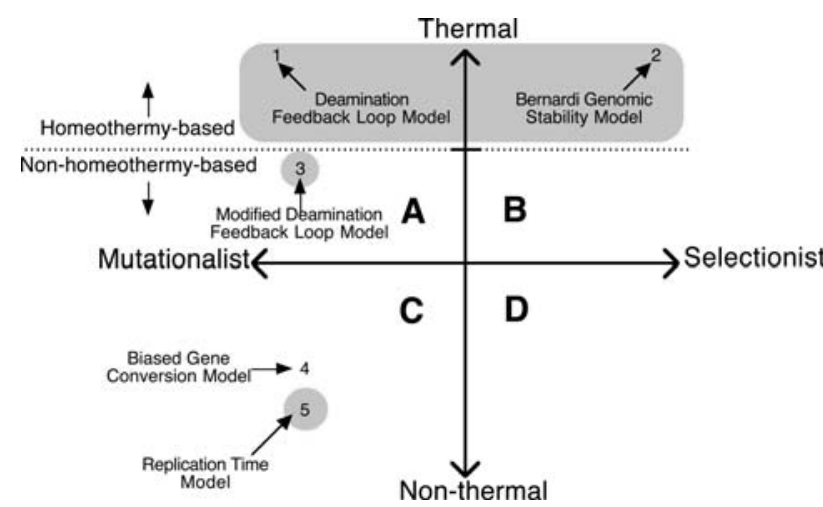

Fig. 1 A unified framework for models of isochore evolution. The axes define mutationalist and selectionist models and emphasize that both types of models can be thermal or nonthermal in nature. Several proposed models are represented on the graph by a number in the relevant position (1. Fryxell and Zuckerkandl 2000; 2. Bernardi 2000; 3. proposed here; 4. Eyre-Walker 1999; 5. Wolfe et al. 1989). This framework emphasizes that models of isochore evolution can be a continuum. The gray areas represent regions of "model space" that can be excluded, based on either our data or that of Eyre-Walker (1992), who examined the replication time model (Wolfe et al. 1989). Rationale for excluding models is detailed in the Discussion section between thermal and nonthermal models (Fig. 1). Bernardi (2000) proposed the prototypical thermal selectionist model, postulating that the selective advantage of GC-rich isochores stems from higher thermodynamic stability of DNA, RNA, and important proteins (e.g., housekeeping proteins) encoded in GC-rich regions- - Bernardi genomic stability" hypothesis. Alternatively, Fryxell and Zuckerkandl (2000) proposed a thermal mutationalist model known as "deamination feedback loop" that postulates a higher rate of cytosine deamination (leading to $\mathrm{C} \rightarrow \mathrm{T}$ transitions) in GC-poor regions at high temperatures, further reducing their GC content and strengthening the feedback loop. This model has a unidirectional GC $\rightarrow$ AT mutational bias, so it is only able to explain the origin of a heterogeneous genome if the homogeneous ancestral genome was GC-rich.

Although there has been substantial focus on thermal models, nonthermal models based on selection or mutation have also been proposed (Eyre-Walker and Hurst 2001; reviewed in Li 1997, pp. 407-411). One of the best-studied models involves GC-biased gene conversion (Galtier et al. 2001), and this model has the benefit of explaining the observed correlation between recombination frequency and GC content (for details see Duret et al. 2002; Eyre-Walker 1993). However, these different models form a continuum where selection and mutation can either generate similar compositional patterns or act in opposition to each other. Placing all of these models into this common framework (Fig. 1) simplifies the examination of different models.

Bernardi (2000) cites several observations as support for the thermal selectionist model. First, homeotherms have strong GC-rich isochore structure while poikilotherms do not. Second, density gradient centrifugation data indicating a number of nonavian reptiles (poikilothermic amniotes) lack the strong GC-rich isochore structure that is characteristic of birds and mammals. Those data suggest the GCrich isochore structure arose independently in birds and mammals from the ancestral "cold-blooded" isochore structure. Third, there is evidence for selection on GC-rich (but not GC-poor) mammalian genes (see Cacciò et al. 1995). Finally, there is population genetic data suggesting that the mammalian MHC genes have been subject to selection favoring $\mathrm{AT} \rightarrow \mathrm{GC}$ mutations or that GC-biased gene conversion has occurred (see Eyre-Walker 1999; note that a "biased gene conversion" model could be a nonthermal mutationalist alternative to models based on selection; Fig. 1). If a thermal model is assumed, combining the data highlighted by Bernardi (2000) and the difficulty reconciling the Fryxell and Zuckerkandl (2000) deamination feedback loop model with a GC-poor ancestral genome creates a framework in which the preponderance of evidence points to a selectionist model. Testing thermal selectionist models requires a rigorous examination of the independent origins of GC-rich isochores in 
birds and mammals, which can be accomplished using comparative genomics.

The apparent phylogenetic support for independent origins of a GC-rich isochore structure in birds and mammals has been proposed to be compelling evidence for a thermal selectionist model (e.g., Bernardi 2000), but two critical assumptions should be examined before accepting such a model. First, the strength of the evidence that the GC-rich isochore structure of birds and mammals was derived independently from a GC-poor ancestral condition should be carefully examined. Second, it is unclear whether increased GC content provides enhanced thermal stability that is advantageous for homeotherms.

Several studies suggest that poikilothermic amniotes have GC-rich isochores. Hughes et al. (1999) reported that thirdcodon-position GC content (GC3) for two poikilothermic amniotes, the Red-eared slider turtle (Trachemys scripta; six genes) and the Nile crocodile (Crocodylus niloticus; ten genes), resembles the GC3 of the chicken. Hamada et al. (2002) expanded this small dataset to 11 genes by adding $\alpha$ globin genes from three snake species, also supporting the existence of GC-rich isochore structure in nonavian reptiles. Hughes et al. (1999) and Hamada et al. (2002) both suggest the common ancestor of amniotes may have had a GC-rich isochore structure, but the limited number of sequences used makes it difficult to view those studies as definitive evidence for the presence of GC-rich isochores in nonavian reptiles.

This study tests critical aspects of the current thermal selectionist model by comparing GC3 for a set of American alligator (Alligator mississippiensis) expressed sequence tag (EST) assemblies to the GC3 of their orthologs in homeothermic amniotes (chicken, human, and mouse) and a poikilothermic vertebrate (the western clawed frog Xenopus tropicalis). We use GC3 variation as a surrogate for isochore structure because previous analyses have shown that GC3 is correlated with intronic GC content (GCi) and the GC content of the larger genomic regions (isochores) in which the genes are located (Bernardi 2000). Our analyses strongly support the existence of a GC-rich isochore structure in the alligator, providing evidence that the shift to GC-rich avian isochores occurred prior to their divergence from crocodilians. This result has profound implications for the set of models that have been used to explain the evolution of isochore structure, and we were able to falsify a subset of these models.

\section{Materials and Methods}

\section{EST Collection and Assembly}

A total of 6732 reads (accession Nos. ES316475ES321899), ranging in length from 76 to 812 nucleotides (mean $484.4 \mathrm{nt}$ ), were obtained from three alligator cDNA libraries using either Applied Biosystems (ABI 377 or ABI 3100 Avant) or Amersham (MegaBACE) automated sequencers. The libraries were from juvenile liver, adult liver, and adult testis, and all reads were assembled essentially as described in Liang et al. (2000), yielding a total of 3064 assemblies. The overall GC content for these assemblies is provided in the supplementary information (Supplementary Fig. 1A), along with the correlation between the overall GC content and the GC3 based upon these EST assemblies (Supplementary Fig. 1B).

EST assemblies were used as tBLASTx (Altschul et al. 1997) queries to search cDNA sequences from the Ensembl database (Birney et al. 2006). Human, mouse, chicken, and frog sequences identified by the alligator query were aligned using ClustalW (Chenna et al. 2003) and alignments that appeared largely anomalous were discarded. The anomalous alignments included those that did not have an ortholog in one of the three amniote species (mouse, chicken, or human) and those that were difficult to align for other reasons (e.g., due to misannotations). The remaining alignments (a total of 366, 98 of which include the frog) were optimized by eye using MacClade (Maddison and Maddison 2002). The segment of the alignment present in all organisms was assigned to a CHARSET. Codon positions were identified and third-position base composition was calculated using PAUP* 4.0b10 (Swofford 1998). Base composition values were extracted from the PAUP logfile using a script and imported into Microsoft Excel.

Because strong correlations have been repeatedly shown between GC3 of protein-coding genes, their introns, and their surrounding DNA (the isochores in which genes are embedded) for both poikilotherm (Bernardi and Bernardi 1991) and homeotherm genomes (Bernardi 2000; Musto et al. 1999), the correlation between GC3 values for orthologous coding sequences is used to study isochore structure. This follows other studies that have examined vertebrate isochore structure (Zoubak et al. 1996) and evolution (Bernardi et al. 1997; Galtier and Mouchiroud 1998). This correlation was examined using the open source of the R software (The R Project for Statistical Computing, http://www.r-project.org/, 1997); the equations were fit in $\mathrm{R}$ using orthogonal regression (Isobe et al. 1990). Lines for the best-fitting equation and unity were added to all plots.

\section{Results}

Histograms of GC3 content for 366 assembled alligator ESTs that could be aligned with their human, mouse, and chicken orthologs revealed a remarkable similarity between the alligator and the homeothermic amniotes 
(Fig. 2). A subset of these assemblies that could also be aligned with their orthologs in the western clawed frog revealed a striking difference in GC3 content between the frog and all amniotes, including the alligator (Fig. 2). When our EST assembly data are viewed in light of the evidence for a correlation between GC3 and the GC content of flanking regions (Bernardi 2000), it suggests that alligators show a degree of genomic heterogeneity due to isochore structure similar to that exhibited by homeothermic amniotes. Only the mouse histogram stands out within the amniotes as having a narrower distribution, and this observation is consistent with previous studies (Bernardi 2000).

Examining the relationships among amniotes in GC3 content revealed a strong positive correlation for all comparisons (Fig. 3), although comparisons of more distantly related organisms showed a lower correlation coefficient than those of more closely related organisms, as expected. GC3 values for amniotes and the frog, used as a representative poikilotherm in previous analyses (e.g., Bernardi 2000), were also correlated (Fig. 4), although the correlation was weaker than that observed within amniotes. The slopes of lines describing the relationship between GC3 values in different amniotes were close to one, with only the human-mouse comparison exceeding unity by a large amount (Fig. 3). In contrast, comparison of either human or alligator with that of the frog revealed a slope even greater than that evident in the human-mouse comparison (Fig. 4 A), making it clear that GC3 values of both homeothermic and poikilothermic amniotes show the same relationship to the frog (Fig. 4B).

\section{Discussion}

These GC3 data indicate that the alligator isochore structure is quite similar to the avian and mammalian isochore structure and different from the amphibian isochore structure. This indicates that strong GC-rich isochore structure, suggested to be present only in homeotherms, actually arose prior to the origin of homeothermy (Fig. 5). Like Duret et al. (2002), we propose that the strong GC-rich isochore structure arose during the origin of amniotes.

The previous work based on a small number of reptilian sequences (Hamada et al. 2002; Hughes et al. 1999) is easy to reconcile with a model in which strong GC-rich isochore structure is a feature of all amniotes and is not limited to homeotherms. In fact, the slope $(m)$ of the line relating chicken and alligator GC3 observed here $(m=0.88)$ is quite similar to that observed by Hughes et al. (1999) in a comparison of Nile crocodile and chicken genes $(m=$ 0.85 ), suggesting that their results were not unreasonably biased by the small number of genes used. Furthermore,
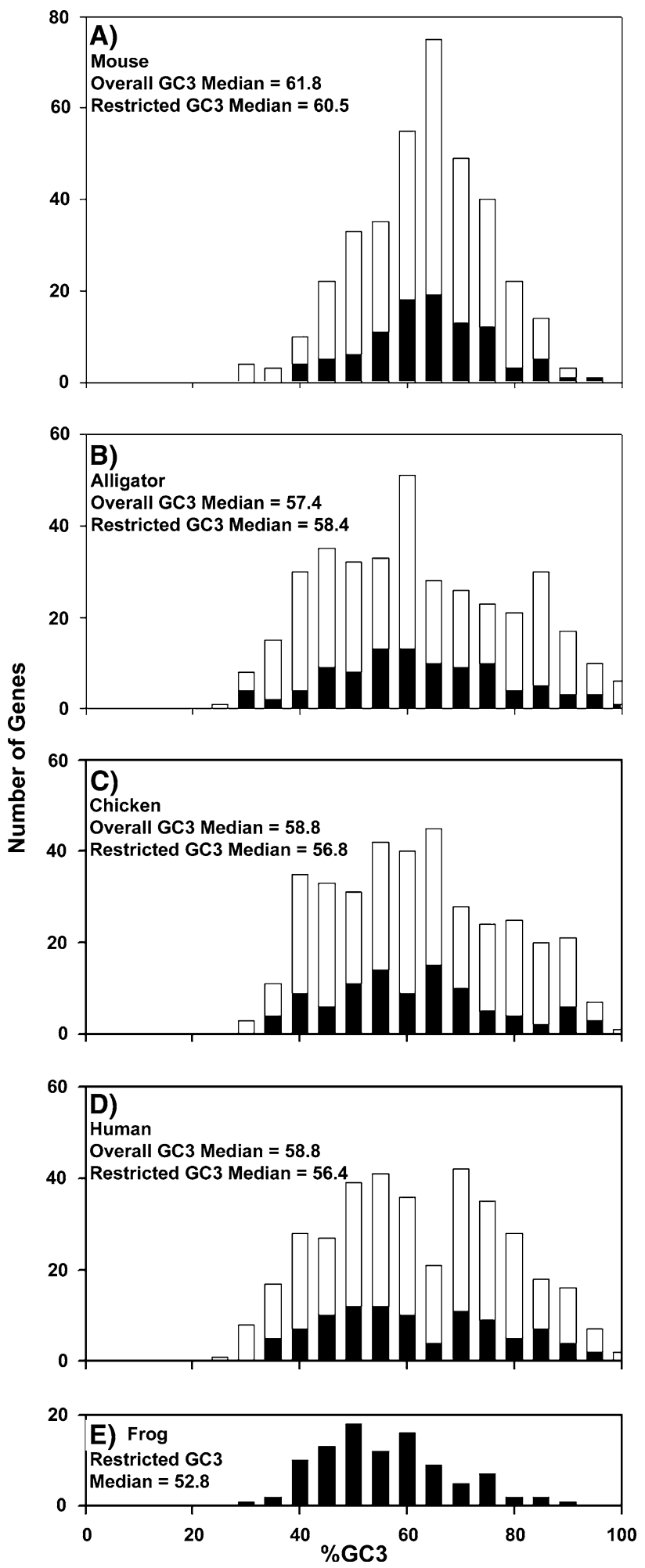

Fig. 2 Histogram showing GC3 content. Genes were placed in bins with a width of $5 \%$. Black bars indicate the subset of 98 alignments that include all four amniotes and the frog (the "restricted set"), while the white portions of the bars indicate the remaining 268 alignments that include the four amniotes (all 366 genes form the "overall set") 
Fig. 3 Correlations among amniotes in GC3 content. The graphs correspond to comparisons between (A) human and mouse, (B) human and chicken, (C) human and alligator, and (D) chicken and alligator. The complete set of 366 genes was included. Comparisons within amniotes that are not presented here can be found in Supplementary Fig. 2, and confidence intervals for the slopes of lines relating GC3 for different organisms can be found in Supplementary Table 1


Fig. 4 Correlations between an amphibian and amniotes in GC3 content. The graphs correspond to comparisons between (A) human and frog and (B) alligator and frog. The smaller 98-gene set was included here. Comparisons between the frog and amniotes that are not presented here can be found in Supplementary Fig. 2, and confidence intervals for the slopes of lines relating GC3 for different organisms can be found in Supplementary Table 1
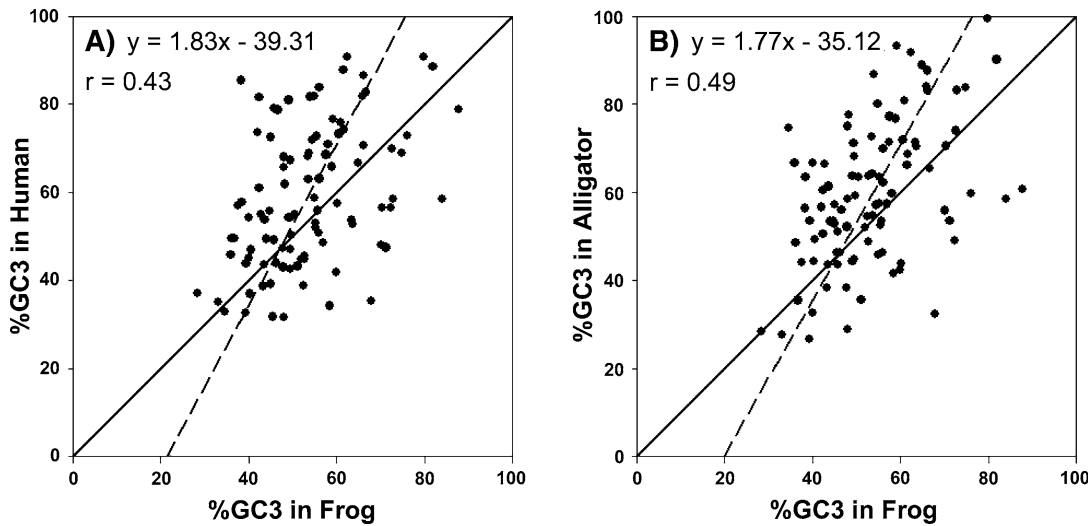

Hughes et al. (1999) observed a similar slope $(m=0.77)$ in another comparison of six red-eared slider turtle and chicken genes. However, the much broader survey of genes in our EST-based study clearly provides greater confidence in the conclusion that GC-rich isochore structure was present in the common ancestor of archosaurs (birds and crocodilians). A logical extension of this conclusion that considers the previous work (e.g., Duret et al. 2002; Hamada et al. 2002; Hughes et al. 1999) would be a model placing the origin of strong GC-rich isochore structure in the common ancestor of all amniotes, but further corroboration of that model will require broader surveys of many additional nonavian reptiles.
The existence of a strong GC-rich isochore structure in the alligator has profound implications for selecting the plausible models for the evolution of isochore structure from the larger set of potential models (Fig. 1). Both the Bernardi (2000) genomic stability model and the deamination feedback loop model (Fryxell and Zuckerkandl 2000) predict that homeotherms will have a strong GCrich isochore structure and that poikilotherms will not (hence the "warm-blooded isochore" terminology typically used in the literature). Since our data indicate a poikilotherm (the alligator) has a strong GC-rich isochore structure similar to homeotherms, both of these models should be considered falsified. However, basing thermal 
models upon the division between homeotherms and poikilotherms may be misleading because there are poikilotherms that are able to maintain a high and stable body temperature similar to homeotherms through behavioral mechanisms (Seebacher and Shine 2004; Seebacher et al. 1999). Thus, the basis for thermal models can reflect changes in thermal biology that occurred during the origin of amniotes (Fig. 5).

The evidence that the origin of GC-rich isochore structure was independent of a shift from poikilothermy to homeothermy (Fig. 5) places an important novel constraint on thermal models of isochore evolution. Nonhomeothermic thermal models must consider multiple body temperature parameters (e.g., both the maximum and mean temperature). For example, a nonhomeothermic version of the Bernardi (2000) genomic stability model might be coupled to the maximum body temperature. In contrast, a nonhomeothermic variant of the deamination feedback loop model is more problematic (noted in Fig. 1 as Modified Deamination Feedback Loop Model), since the accumulation of thermally driven mutations would be expected to reflect the mean body temperature. Thus, less change from the ancestral state (high GC) is predicted in poikilotherms, which should lead to higher GC content in alligators relative to homeotherms. Our data contradict this prediction because we found that low GC3 genes in chicken also have low GC3 in the alligator (Fig. 3D).

Genomic comparisons similar to this study that focus on organisms from different thermal environments and with different mechanisms for physiologic and behavioral thermoregulation may further constrain thermal models of isochore evolution. For example, the tropical pufferfish Tetraodon nigroviridis has an approximately $4 \%$ higher mean GC content than its temperate relative Fugu rubripes (Jabbari and Bernardi 2004), which is consistent with a nonhomeothermic thermal model of isochore evolution. However, when these types of comparisons are conducted on a large scale, it will be important to acknowledge that isochore structure may be more reflective of the historical environment of organisms' ancestors than the present-day environment. In fact, there are aspects of evolutionary history that may have an impact on the present study, like the possibility that the ancestors of extant crocodilians were homeotherms (Seymour et al. 2004; but see Hillenius and Ruben 2004). Thus, the evaluation of genomic data should be conducted in a phylogenetic framework that also considers the paleoenvironment.

The phylogenetic evidence suggesting a single origin of the GC-rich isochore structure of amniotes indicates that nonthermal models (regions $\mathrm{C}$ and D of Fig. 1) should receive additional attention. It is easy to imagine specific genetic changes at the ancestor of modern amniotes that might establish either a mutational bias or a selective

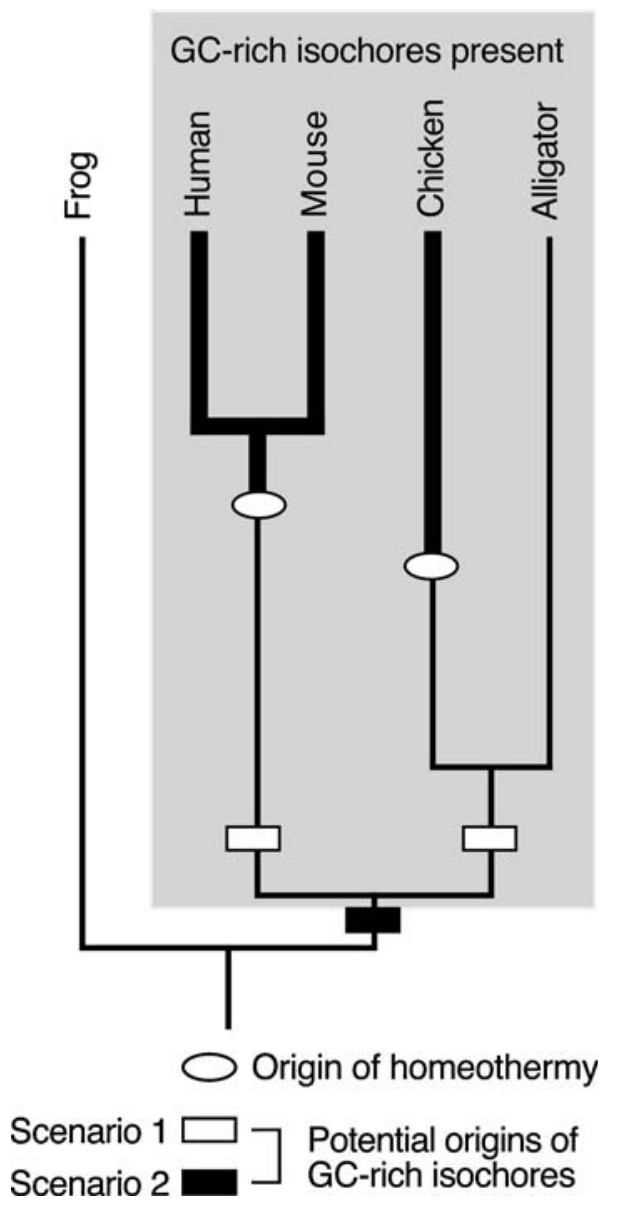

Fig. 5 Strong GC-rich isochore structure appears to unite amniotes. Tetrapod phylogeny showing multiple origins of homeothermy and the distribution of strong GC-rich isochore structure (indicated using the gray region). The most parsimonious reconstruction of character states is a single origin of strong GC-rich isochore structure at the base of the amniotes (scenario 1). However, the limited taxon sampling available does not allow us to exclude two independent origins of strong GC-rich isochore structure (scenario 2), one in mammals and one in archosaurs (birds and crocodilians). Neither scenario is consistent with a correlation between the origin of homeothermy and the origin of GC-rich isochore structure. The ancestral GC-poor state for tetrapod isochores reflects information on amphibians (e.g., our data) and other outgroup taxa (e.g., Bernardi 2000). Branch lengths reflect divergence times based on the fossil record (summarized in Benton and Donoghue 2007)

environment that would generate a genomic structure uniting the amniotes (like the strong GC-rich isochore structure). For example, a change in chromatin proteins could lead either to regional mutational biases or to selection that favors GC-rich alleles in genic regions. The accumulation of evidence suggesting that isochore evolution may not have a thermal basis should open the field to novel routes of inquiry.

Both thermal and nonthermal models should be examined using a historical framework. For example, the strong GC-rich isochore structure of mammals has been degrading 
(Duret et al. 2002; Webster et al. 2006), suggesting that analyses of mammalian genomes alone may not reveal the processes that led to the strong GC-rich isochore structure of amniotes. This is illustrated by Eyre-Walker's (1992) test of the Wolfe et al. (1989) replication time model (a nonthermal mutationalist model). The replication time model postulates that genes replicating early in $\mathrm{S}$ phase of the cell cycle show different mutational patterns (e.g., $\mathrm{AT} \rightarrow \mathrm{GC}$ ) than those replicating late in $\mathrm{S}$ phase. Using 44 mammalian (primate and rodent) genes, Eyre-Walker (1992) did not find the correlation between replication time and GC content expected if this model were correct (Fig. 1). Although this result is compelling, it remains possible that birds will exhibit this correlation because GCrich isochore structure appears to be strengthening in birds (Webster et al. 2006). If so, the absence of this correlation in mammals might reflect the mechanistic basis for the degradation of GC-rich isochores reported by Duret et al. (2002). Thus, a broader phylogenetic and historical perspective, including data from birds and other reptiles, would greatly increase the power of such analyses.

Our analysis of alligator EST data provides a framework that can be expanded by the acquisition of EST or genomic sequence data from additional organisms, especially reptiles. The present work provides a conclusive answer regarding the existence of a strong GC-rich isochore structure in a poikilotherm. However, a broader survey would have the potential to either further constrain the set of credible models for isochore evolution or provide novel information necessary to define additional models that are better able to explain isochore evolution.

Acknowledgments We are grateful to the members of the BraunKimball lab group for helpful discussion and to Holly Kindsvater and Mike McCoy for help with R and statistical advice. This work was supported in part by grants to Y.K. and T.I. (Grant-in-Aid for Scientific Research from the Ministry of Education, Science, Sports and Culture of Japan and grants from the Ministry of Environment, Japan) and to E.L.B. and L.J.G. (University of Florida Opportunity Fund).

\section{References}

Altschul SF, Madden TL, Schaffer AA, Zhang J, Zhang Z, Miller W, Lipman DJ (1997) Gapped BLAST and PSI-BLAST: a new generation of protein database search programs. Nucleic Acids Res 25:3389-3402

Benton MJ, Donoghue PC (2007) Paleontological evidence to date the tree of life. Mol Biol Evol 24:26-53

Bernardi G (1995) The human genome: organization and evolutionary history. Annu Rev Genet 29:445-447

Bernardi G (2000) Isochores and the evolutionary genomics of vertebrates. Gene 241:3-17

Bernardi G, Bernardi G (1991) Compositional properties of nuclear genes from cold-blooded vertebrates. J Mol Evol 33:57-67

Bernardi G, Hughes S, Mouchiroud D (1997) The major compositional transitions in the vertebrate genome. J Mol Evol 44:44-51
Birney E, Andrews D, Caccamo M, et al. (2006) Ensembl 2006. Nucleic Acids Res 34:556-561

Cacciò S, Zoubak S, D’Onofrio G, Bernardi G (1995) Nonrandom frequency patterns of synonymous substitutions in homologous mammalian genes. J Mol Evol 40:280-292

Cacciò S, Jabbari K, Matassi G, Guermonprez F, Desgres J, Bernardi G (1997) Methylation patterns in the isochores of vertebrate genomes. Gene 205:119-124

Chenna R, Sugawara H, Koike T, Lopez R, Gibson TJ, Higgins DG, Thompson JD (2003) Multiple sequence alignment with the Clustal series of programs. Nucleic Acids Res 31:3497-3500

Duret L, Semon M, Piganeau G, Mouchiroud D, Galtier N (2002) Vanishing GC-rich isochores in mammalian genomes. Genetics 162:1837-1847

Eyre-Walker A (1992) Evidence that both $\mathrm{G}+\mathrm{C}$ rich and $\mathrm{G}+\mathrm{C}$ poor isochores are replicated early and late in the cell cycle. Nucleic Acids Res 20:1497-1501

Eyre-Walker A (1993) Recombination and mammalian genome evolution. Proc Biol Sci 252:237-243

Eyre-Walker A (1999) Evidence of selection on silent site base composition in mammals: potential implications for the evolution of isochores and junk DNA. Genetics 152:675-683

Eyre-Walker A, Hurst LD (2001) The evolution of isochores. Nat Rev Genet 2:549-555

Fryxell KJ, Zuckerkandl E (2000) Cytosine deamination plays a primary role in the evolution of mammalian isochores. Mol Biol Evol 17:1371-1383

Galtier N, Mouchiroud D (1998) Isochore evolution in mammals: a human-like ancestral structure. Genetics 150:1577-1584

Galtier N, Piganeau G, Mouchiroud D, Duret L (2001) GC-content evolution in mammalian genomes: the biased gene conversion hypothesis. Genetics 159:907-911

Hackenberg M, Bernaola-Galvan P, Carpena P, Oliver JL (2005) The biased distribution of Alus in human isochores might be driven by recombination. J Mol Evol 60:365-377

Hamada K, Horiike T, Kanaya S, Nakamura H, Ota H, Yatogo T, Okada K, Nakamura H, Shinozawa T (2002) Changes in body temperature pattern in vertebrates do not influence the codon usages of $\alpha$-globin genes. Genes Genet Syst 77:197-207

Hillenius WJ, Ruben JA (2004) Getting warmer, getting colder: Reconstructing crocodylomorph physiology. Physiol Biochem Zool 77:1068-1072

Hughes S, Zelus D, Mouchiroud D (1999) Warm-blooded isochore structure in Nile crocodile and turtle. Mol Biol Evol 16:15211527

Isobe T, Feigelson ED, Akritas MG, Babu GJ (1990) Linear regression in astronomy. Astrophys J 364:104-113

Jabbari K, Bernardi G (2004) Body temperature and evolutionary genomics of vertebrates: a lesson from the genomes of Takifugu rubripes and Tetraodon nigroviridis. Gene 333:179-188

Li WH (1997) Molecular Evolution. Sinauer Associates, Sunderland, MA, pp 407-411

Liang F, Holt I, Pertea G, Karamycheva S, Salzberg SL, Quackenbush J (2000) An optimized protocol for analysis of EST sequences. Nucleic Acids Res 28:3657-3665

Maddison D, Maddison W (2002) MacClade. Sinauer Associates, Sunderland, MA

Musto H, Romero H, Zavala A, Bernardi G (1999) Compositional correlations in the chicken genome. J Mol Evol 49:325-329

Saccone S, Cacciò S, Perani P, Andreozzi L, Rapisarda A, Motta S, Bernardi G (1997) Compositional mapping of mouse chromosomes and identification of the gene-rich regions. Chromosome Res 5:293-300

Seebacher F, Shine R (2004) Evaluating thermoregulation in reptiles: the fallacy of the inappropriately applied method. Physiol Biochem Zool 77:688-695 
Seebacher F, Grigg GC, Beard LA (1999) Crocodiles as dinosaurs: behavioural thermoregulation in very large ectotherms leads to high and stable body temperatures. J Exp Biol 202:77-86

Seymour RS, Bennett-Stamper CL, Johnston SD, Carrier DR, Grigg GC (2004) Evidence for endothermic ancestors of crocodiles at the stem of archosaur evolution. Physiol Biochem Zool 77:10511067

Swofford D (1998) PAUP*: phylogenetic analysis using parsimony (*and other methods), ver. 4. Sinauer Associates, Sunderland, MA

Varriale A, Bernardi G (2006) DNA methylation in reptiles. Gene 385:122-127
Webster MT, Axelsson E, Ellegren H (2006) Strong regional biases in nucleotide substitution in the chicken genome. Mol Biol Evol 23:1203-1216

Wolfe KH, Sharp PM, Li WH (1989) Mutation rates differ among regions of mammalian genome. Nature 337:283-285

Zhang CT, Wang J, Zhang R (2001) A novel method to calculate the $\mathrm{G}+\mathrm{C}$ content of genomic DNA sequences. J Biomol Struct Dyn 19:333-341

Zoubak S, Clay O, Bernardi G (1996) The gene distribution of the human genome. Gene 174:95-102 